\title{
Interactions of silica nanoparticles with lung epithelial cells and the association to flotillins
}

\author{
Jennifer Kasper • Maria I. Hermanns • Christoph Bantz • \\ Olga Koshkina - Thomas Lang - Michael Maskos • \\ Christine Pohl · Ronald E. Unger · C. James Kirkpatrick
}

Received: 2 March 2012 / Accepted: 16 May 2012/ Published online: 6 June 2012

(C) The Author(s) 2012. This article is published with open access at Springerlink.com

\begin{abstract}
Amorphous silica nanoparticles (aSNPs) gain increasing popularity for industrial and therapeutic claims. The lung with its surface area of $100-140 \mathrm{~m}^{2}$ displays an ideal target for therapeutic approaches, but it represents also a serious area of attack for harmful nanomaterials. The exact nature of the cytotoxic effects of NPs is still unknown. Furthermore, cellular pathways and the destiny of internalized NPs are still poorly understood. Therefore, we examined the cytotoxicity (MTS, LDH) and inflammatory responses (IL-8) for different-sized aSNPs (30, 70, $300 \mathrm{~nm}$ ) on our lung epithelial cells line NCI H441 and endothelial cell line ISO-HAS-1. Additionally, colocalization studies have been conducted via immunofluorescence staining for flotillin-1- and flotillin-2-bearing endocytic vesicles. Subsequently, the relevance of flotillins concerning the viability of aSNP-exposed epithelial cells has been evaluated using flotillin-1/2 depleted cells (siRNA). This study reveals the relevance of the nanoparticle size
\end{abstract}

J. Kasper $(\bowtie)$ C C. Pohl · R. E. Unger · C. J. Kirkpatrick Institute of Pathology, University Medical Centre,

Mainz, Germany

e-mail: kasperj@uni-mainz.de

M. I. Hermanns

ikfe $\mathrm{GmbH}$, Institut für klinische Forschung und Endwicklung,

Mainz, Germany

C. Bantz $\cdot$ M. Maskos

Institute of Physical Chemistry, Johannes Gutenberg University

Mainz, Mainz, Germany

O. Koshkina · T. Lang · M. Maskos

BAM Federal Institute for Materials Research and Testing,

Berlin, Germany

M. Maskos

Institut für Mikrotechnik, Mainz, Germany regarding cytotoxicity (MTS, LDH) and inflammatory responses (IL-8), whereat the smaller the size of the nanoparticle is, the more harmful are the effects. All different aSNP sizes have been incorporated in flotillin-1- and flotillin-2labelled vesicles in lung epithelial and endothelial cells, which display a marker for late endosomal or lysosomal structures and appear to exhibit a clathrin- or caveolae-independent mode of endocytosis. Flotillin-depleted H441 showed a clearly decreased uptake of aSNPs. Additionally, the viability of aSNP-exposed cells was reduced in these cells. These findings indicate a contribution of flotillins in as yet unknown (clathrin or caveolae-independent) endocytosis mechanisms and (or) endosomal storage.

Keywords Silica nanoparticles .

Alveolar-capillary barrier · Lung epithelial cells .

Endothelial cells · Endocytosis · Flotillin-1 · Flotillin-2 .

Cytotoxicity $\cdot$ Inflammatory response

\begin{tabular}{ll}
\multicolumn{2}{l}{ Abbreviations } \\
ANOVA & Analysis of variance \\
aSNP & Amorphous silica nanoparticles \\
DLS & Dynamic light scattering \\
EC & Endothelial cells \\
Flot1 & Flotillin-1 \\
Flot2 & Flotillin-2 \\
IF & Immunofluorescence \\
NP & Nanoparticle \\
PBSA & Phosphate buffered saline with $1 \%$ bovine \\
& serum albumin \\
Pen/Strep & Penicillin/streptomycin \\
RFU & Relative fluorescent unit \\
SNPs & Silica nanoparticles \\
F12 & Flotillin-1/-2 depletion \\
Neg & Negative siRNA
\end{tabular}




\section{Background}

Nanotechnology has made tremendous progress in the last decade concerning industrial applications of nanoparticles (NPs) due to their different features compared to the same material in bulk form (Service 2004). Almost any material can be used to produce engineered nanoparticles for industrial purposes (carbon: lubricants, coatings; titanium dioxide: toothpaste, white paints, sunscreens; silver: textile coating with antimicrobial properties; Ellsworth et al. 2000; Institute of Nanotechnology 2011). In particular, amorphous silica nanoparticles (aSNPs) are widely used for industrial applications. aSNPs are processed in paints and coatings, photovoltaic systems, tyre compounds or electrical and thermal insulation material (AEROSIL ${ }^{\circledR}$, EVONIK Industries, http://www.aerosil.com).

They are also added to products to which humans have direct exposure, for example, cosmetics, toothpaste or even powdered food ingredients to avoid caking (Waters et al. 2009; Borm et al. 2006). Furthermore, aSNPs among others are considered to be a promising tool to develop drug and gene carriers purposed for diagnostic and therapeutical approaches (Liu et al. 2011). In general, nanoparticles are increasingly gaining attention in biomedical research for pulmonary delivery of, that is, insulin or calcitonin (Kawashima et al. 1999; Yamamoto et al. 2005; Zhang et al. 2001). Recent research activity involves the development of nanovehicles loaded with, for example, anti-asthmatic drugs (Surti et al. 2008). On the one hand, the lung is an ideal target organ for drug and gene delivery via nanoparticles for both systemic and local applications (asthma, COPD, cystic fibrosis) due to its enormous surface area of ca. $140 \mathrm{~m}^{2}$ and the reduced first-pass metabolism, by which the concentration of an applied drug is drastically reduced before reaching the systemic circulation, not to mention the targeted organ (Hoet et al. 2004; Bailey and Berkland 2009). On the other hand, these characteristics make the lung an ideal target site for accidently inhaled hazardous nanoparticles. Hence, attention must be given to possible health hazards of inhalation of NPs destined for industrial or biomedical applications or targets. The attention should not only be focused on cytotoxic effects. Inhaled nanoparticles may cause unwanted inflammatory responses. What is not known are the exact nature of the cytotoxic effects. Furthermore, cellular pathways of internalized NPs and their eventual distribution and localization within the cells are generally not known. It is known that nanoparticle deposition in the peripheral lung appears to be size dependent. Nanosized particulate matter more likely reaches the deep lung compared to micronsized particles (Bailey and Berkland 2009). Furthermore, the deposition efficiencies of different-sized nanoparticles vary for the different regions of the lung (Oberdorster et al. 2005). The size of a nanoparticle is regarded as a pivotal characteristic factor and closely correlates with its surface area. The surface parameter is regarded as a prevailing factor with respect to the reactivity and cytotoxicity of the nanomaterials (Kreyling et al. 2010).

Numerous studies have shed some light on the toxic effects of silica nanoparticles in general (Sun et al. 2011; Nabeshi et al. 2011) or focused on size-dependent toxic effects of aSNPs ( $\mathrm{Li}$ et al. 2011; Napierska et al. 2009). However, size-dependent inflammatory responses caused by silica nanoparticles are still poorly understood and may display a more sensitive reaction compared to the standard cytotoxicity tests. The goal of this study was to further characterize the toxic effects and uptake of aSNPs. The cytotoxic effects of different-sized aSNPs, which were exposed to the human lung epithelial cell line NCI H441 (H441) and the microvascular endothelial cell line ISOHAS-1, were examined using the MTS assay, a test for cell viability and the LDH assay, an assay for membrane integrity. In addition, studies were done to investigate the inflammatory responses (IL-8 release) of $\mathrm{H} 441$ and ISOHAS-1 following treatment with different-sized aSNPs. Furthermore, cellular uptake and location of different-sized aSNPs in H441 and ISO-HAS-1 cells was visualized by means of colocalization experiments via immunofluorescent staining of flotillin-1 and flotillin-2, which display a marker for late endosomal or lysosomal structures and appear to exhibit a clathrin- or caveolae-independent mode of endocytosis (Glebov et al. 2006). Finally, we examined the importance of flotillin-1 and flotillin-2 on cytotoxic effects and uptake behaviour of aSNPs in flotillin-containing and flotillin-1- and flotillin-2-depleted H441 generated by siRNA transfection.

\section{Materials and methods}

\section{Nanoparticle characterization}

Sicastar Red are amorphous silica nanoparticles in aqueous dispersion with nominal diameters of 30,70 and $300 \mathrm{~nm}$. The particles are loaded with a fluorophore, namely Rhodamine $\mathrm{B}$, which is covalently attached to the $\mathrm{SiO}_{2}$ matrix. Sicastar Red is commercially available from micromod Partikeltechnologie GmbH (www.micromod.de). The samples were characterized by angular-dependent dynamic light scattering measurements as described previously (Kasper et al. 2011).

In pure aqueous solution, Sicastar Red particles with the nominal diameter of $30 \mathrm{~nm}$ showed a hydrodynamic radius $\mathrm{R}_{\mathrm{h}}$ of $12.6 \mathrm{~nm}$ (corresponding diameter: $25.2 \mathrm{~nm}$; see Table 1), indicating that the particles are in a non-aggregated primary particle state. However, in salt-containing 
Table 1 Characterization of fluorescence-labelled, silica-based nanoparticles

\begin{tabular}{|c|c|c|c|c|c|c|}
\hline & \multicolumn{2}{|l|}{ Water } & \multicolumn{2}{|l|}{ PBS } & \multicolumn{2}{|l|}{ RPMI } \\
\hline & $\mathrm{R}_{\mathrm{h}} / \mathrm{nm}$ & $\mu_{2}\left(90^{\circ}\right)$ & $\mathrm{R}_{\mathrm{h}} / \mathrm{nm}$ & $\mu_{2}\left(90^{\circ}\right)$ & $\mathrm{R}_{\mathrm{h}} / \mathrm{nm}$ & $\mu_{2}\left(90^{\circ}\right)$ \\
\hline Sicastar Red, $30 \mathrm{~nm}$ & 12.6 & 0.10 & 66.2 & 0.16 & 58.1 & 0.17 \\
\hline Sicastar Red, $70 \mathrm{~nm}$ & 33.2 & 0.06 & 34.1 & 0.03 & 31.6 & 0.03 \\
\hline Sicastar Red, $300 \mathrm{~nm}$ & 157 & 0.02 & 151 & 0.01 & 151 & 0.04 \\
\hline
\end{tabular}

Hydrodynamic radii ( $\mathrm{Rh}$, with $\mu_{2}$ values as an estimate for the polydispersity) in different aqueous solutions (Milli-Q water, phosphate buffered saline (PBS, without $\mathrm{Ca}^{2+}$ and $\mathrm{Mg}^{2+}$ ) and serum-free cell culture medium RPMI) were obtained by dynamic light scattering (angular-dependent measurements)

buffer solution such as PBS and in serum-free cell culture medium RPMI, these dispersions got destabilized and the particles partly agglomerated: The average hydrodynamic radius in PBS is around 66 and $58 \mathrm{~nm}$ in RPMI. Simultaneously, the $\mu_{2}$ values, representing the width of the particle size distribution and thus being a measure for the samples polydispersity, also increased. This leads to the assumption that-despite the increased average hydrodynamic radii-there is still some non-agglomerated material left in the dispersion.

Contrary to this observation, Sicastar Red with nominal diameters of 70 and $300 \mathrm{~nm}$ showed sizes similar to the nominal ones in all aqueous media (i.e. in RPMI: $31.6 \mathrm{~nm}$ for the $70 \mathrm{~nm}$ aSNP and $151 \mathrm{~nm}$ for the $300 \mathrm{~nm}$ aSNP). Also, the size distributions of these two particles are very narrow in all cases, even close to true monodispersity, that is, $\mu_{2}=0.03$ for Sicastar $70 \mathrm{~nm}$ and $\mu_{2}=0.04$ for Sicastar $300 \mathrm{~nm}$, both in RPMI (samples with $\mu_{2}$ values $<0.05$ are generally considered as monodisperse). Thus, the tendency to form aggregates is limited to the smallest particles in this study. Figure 1 depicts the Sicastar nanoparticles in dry state via TEM (transmission electron microscopy). TEM revealed similar radii of the aSNPs as obtained via DLS
(Sicastar 30: $R=11.7 \pm 1.7 \mathrm{~nm}( \pm 14.3 \%)$, Sicastar 70: $R=32.3 \pm 4.1 \mathrm{~nm}( \pm 12.6 \%)$, Sicastar 300: $R=163.7 \pm$ $11.2 \mathrm{~nm}( \pm 6.9 \%)$. Scale bar $=100 \mathrm{~nm})$.

Cell culture and nanoparticle exposure

\section{Cell culture}

ISO-HAS-1 (human microvascular endothelial cell line, (Masuzawa et al. 1999; Unger et al. 2002)) and NCI H441 (human lung adenocarcinoma cell line, purchased from ATCC, ATCC-HTB-174, Promochem, Wesel, Germany) were kept in RPMI 1640 supplemented with $10 \%$ FCS (foetal calf serum), $1 \% \mathrm{P} / \mathrm{S}$ (Penicillin/Streptomycin). ISO-HAS- 1 and H441 were passaged every third day in a dilution of $1: 3$ until passage 50 and 35 , respectively.

\section{Monocultures in experimental procedures}

Prior to seeding cells, the 96-well plates (TPP, Switzerland) or 8-well $\mu$-slides (ibidi) were coated with 50/300 $\mu \mathrm{l}$ fibronectin for $1 \mathrm{~h}$ at $37{ }^{\circ} \mathrm{C}(5 \mu \mathrm{g} / \mathrm{ml}$, Roche Diagnostics, Mannheim). The cells were seeded (ISO-HAS-1: $1.6 \times 10^{4}$
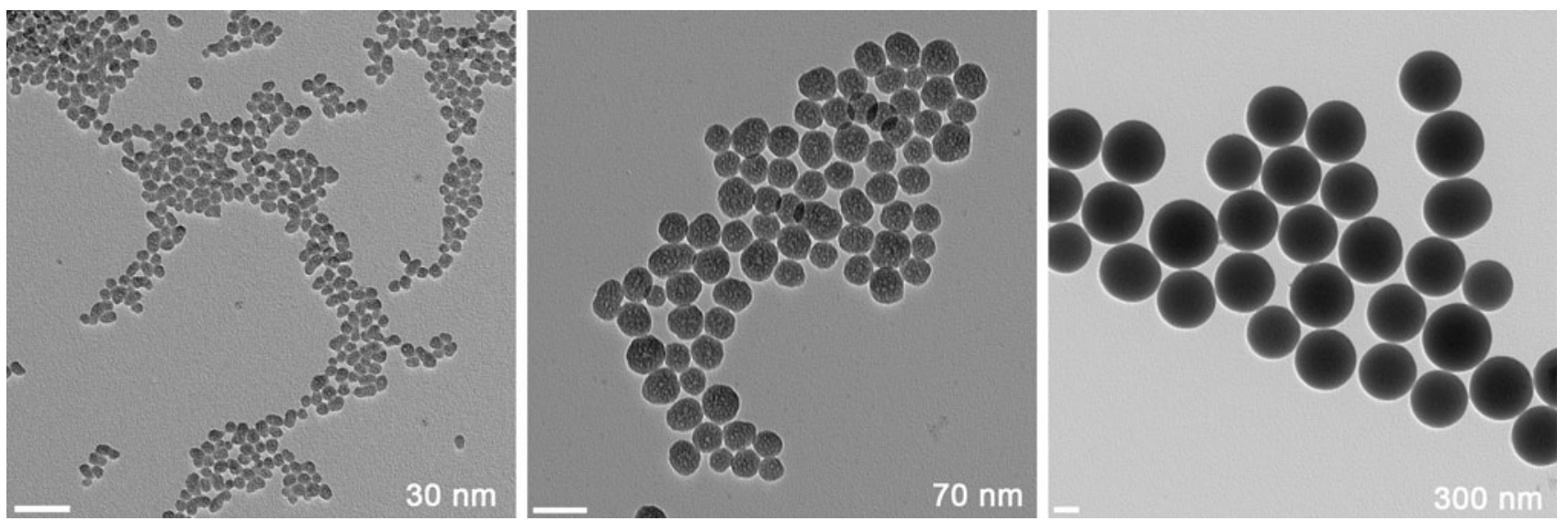

Fig. 1 Electron microscopical image of the Sicastar nanoparticles in dry state. The fact that the size of the nanoparticles in the dry state $\left(D_{T E M}\right)$ was nearly the same as in solution indicates that the effect of particle shrinking during the preparation of the samples for TEM is minimal. Sicastar 30: $R=11.7 \pm 1.7 \mathrm{~nm}( \pm 14.3 \%)$, Sicastar 70: $R=32.3 \pm 4.1 \mathrm{~nm}( \pm 12.6 \%)$, Sicastar 300: $R=163.7 \pm 11.2 \mathrm{~nm}$ $( \pm 6.9 \%)$. Scale bar $100 \mathrm{~nm}$ 
cells/well, H441: $3.2 \times 10^{4}$ cells/well) from a confluent culture flask on 96-well plates in RPMI 1640 medium (Gibco) with L-glutamine supplemented with $10 \%$ FCS and Pen/Strep (100 U/100 $\mu \mathrm{g} / \mathrm{ml})$ and cultivated at $37{ }^{\circ} \mathrm{C}$, $5 \% \mathrm{CO}_{2}$ for $24 \mathrm{~h}$ prior to NP exposure to a confluent cell layer.

\section{Nanoparticle application on cell culture}

To prevent nanoparticle aggregation, pre-dilutions of the NP dispersions were prepared in pure water (Braun ad injectabilia, Braun Melsungen AG, Melsungen). Due to nanoparticle aggregation in serum-containing medium, serum-free medium was used during 4-h exposure. All dilutions were applied 1:10 in serum-free medium to the cells (96 well and transwells: $10 \mu \mathrm{lN}$ dispersion $+90 \mu \mathrm{l}$ serum-free medium, and ibidi wells: $30 \mu \mathrm{l}$ NP dispersion $+270 \mu \mathrm{l}$ serum-free medium). To study cytotoxicity and inflammatory responses, an exposure time of 4 and $4 \mathrm{~h} /$ $20 \mathrm{~h}$ (after 4-h incubation, cells were washed twice with serum-free medium and further cultivated for 20 -h period under normal culture condition) was chosen. For colocalization studies, an exposure time of $20 \mathrm{~min}, 4$ and $4 \mathrm{~h} / 20 \mathrm{~h}$ was chosen.

\section{Cytotoxicity}

The viability of the cells was determined using the CellTiter $96^{\circledR}$ AQueous One Solution Cell Proliferation Assay (MTS, Promega, G3582). After nanoparticle incubation, medium was removed and cells were washed twice with PBS to remove nanoparticle remnants, which can interfere with the MTS reagent. The MTS reagent (MTS stock solution mixed with medium in a ratio of $1: 10$ ) was applied on the cell layer for $45 \mathrm{~min}$ and transferred into a new plate to measure OD at $492 \mathrm{~nm}$.

\section{Membrane integrity}

$25 \mu \mathrm{l}$ of the supernatant, collected from nanoparticle exposed H441 and ISO-HAS-1, was used in the LDH CytoTox $96^{\circledR}$ Non-Radioactive Cytotoxicity Assay (Promega, G1780) to determine lactate dehydrogenase (LDH) release following membrane disruption. To avoid falsepositive results in the LDH assay after 4-h exposure, the percentage due to aSNP interferences with the dye has been subtracted from the measurements (i.e. Sicastar Red, $30 \mathrm{~nm}$ with $600 \mu \mathrm{g} / \mathrm{ml}: 9.8 \pm 1.9 \%$ of lysis control).

\section{Inflammatory responses}

The supernatant of nanoparticle exposed H441 was taken to determine IL-8 release via ELISA (DuoSet R\&D,
DY208) following the manufacturer's recommendations. Immunofluorescence (IF) was performed to label endosomal marker proteins flotillin-1 and flotillin-2 (BD, 610821, 610383). After nanoparticle exposure, cells were fixed with methanol/ethanol (endosomal markers) in a ratio of 2:1 for $15 \mathrm{~min}$ at room temperature. After fixation, cells were incubated with primary antibody diluted in $1 \%$ PBSA (phosphate buffered saline with $1 \%$ bovine serum albumin) over night at $4{ }^{\circ} \mathrm{C}$. After three washing steps with PBS, cells were incubated with secondary antibody (Alexa Fluor 488 ) for $1 \mathrm{~h}$ at room temperature. Subsequently, cells were washed three times with PBS and nuclei were stained with Hoechst 33342 (Molecular Probes) for 5 min and washed three times. Finally, ibidi $\mu$-slides were mounted with ibidi mounting medium (ibidi, Martinsried).

\section{Transfection of H441 with siRNA}

siRNAs were purchased from Ambion (Applied Biosystems: Flotillin-1: s19914 (GCAUCAGUGUGGUUAGCU Att) and Flotillin-2: s5286 (GACUAUAAACAGUACGU GUtt). To prepare the siRNA transfection mixture, siRNA was diluted in serum-free medium and added to the same volume of Gencarrier-1-mix (Gencarrier-1 stock solution diluted 1:25 in serum-free medium) and incubated for 30 min at RT. The final siRNA concentration in the well was $50 \mathrm{nM}$. The transfection was conducted in parallel to the seeding of cells on tissue culture plastic (TPP). $5 \times 10^{5}$ (6-well plates) or $3 \times 10^{4}$ (96-well plates) cells were seeded in $800 \mu \mathrm{l}$ (6-well plates) or $40 \mu \mathrm{l}$ (96-well plates) of low-serum medium (RPMI with $1 \%$ FCS) into a well and siRNA transfection mixture $(200 \mu \mathrm{l}$ for 6 -well plates and $10 \mu \mathrm{l}$ for 96-well plates) has been applied to the cells dropwise and mixed thoroughly. After $7 \mathrm{~h}$ of incubation at $37^{\circ} \mathrm{C}$ and $5 \% \mathrm{CO}_{2}, 1 \mathrm{ml}(50 \mu \mathrm{l})$ of high-serum medium (20\% FCS) was added and the cell culture has been continued for further $17 \mathrm{~h}$. After $24 \mathrm{~h}$, the transfection medium was replaced by normal culture medium and the cells were cultured for further $48-72 \mathrm{~h}$ to achieve the highest siRNAinhibition efficiency. Inhibition efficiency of the siRNA was checked via real-time PCR (Power SYBR ${ }^{\circledR}$ Green PCR Master Mix, Applied Biosystems, 436765, Real time PCR machine: 7300 Real Time PCR System, AB). RNA isolation was conducted by means of the RNeasy Mini Kit (Qiagen, 74104) following the manufacturer's protocol. Reverse transcription of the RNA was performed using the Omniscript Reverse Transcriptase-System (Qiagen, 205113).

Statistical analysis

From several independent measurements, means and standard deviations were calculated. Analyses are shown as 
mean $\pm \mathrm{SD}$ from at least three separate experiments. Testing for significant differences between means was carried out using one-way ANOVA and Dunnett's multiple comparison test at a probability of error of $5 \%(*), 1 \%$ $(* *)$ and $0.1 \%(* * *)$ or two-way ANOVA with Bonferroni's post-test.

\section{Results}

Cytotoxicity and inflammatory responses

Figure 2a depicts the viability of H441 and ISO-HAS-1 in conventional monoculture after exposure to different-sized aSNPs (Sicastar Red with nominal diameters of 30, 70 and $300 \mathrm{~nm}$, as stated by the supplier) with an incubation period of $4 \mathrm{~h}$ at a concentration range of $6,60,150,300,600 \mu \mathrm{g} / \mathrm{ml}$ (which is consistent with $6.9 \times 10^{9} ; 6.9 \times 10^{10} ; 1.8 \times 10^{11}$; $\left.3.6 \times 10^{11} ; 6.9 \times 10^{11} \mu \mathrm{g} / \mathrm{cm}^{2}\right)$ in a volume of $100 \mu \mathrm{l} / \mathrm{well}$. For both cell lines, significant differences in cytotoxic effects between the three different-sized aSNP were observed (concentration of NPs $150 \mu \mathrm{g} / \mathrm{ml}$ ). $30 \mathrm{~nm}$-sized aSNPs caused a reduced viability in $\mathrm{H} 441(77 \pm 13 \%$ of untreated control) compared to the larger-sized aSNPs of 70 and $300 \mathrm{~nm}$, which did not show a significant cytotoxic effect (70 nm: $90 \pm 15 \%, 300 \mathrm{~nm}: 96 \pm 10 \%$ of untreated control). Higher concentrations ( 300 and $600 \mu \mathrm{g} / \mathrm{ml}$ ) illustrate a clearly increased toxicity of the smaller-sized aSNPs of $30 \mathrm{~nm}(300 \mu \mathrm{g} / \mathrm{ml}: 23 \pm 6 \%, 600 \mu \mathrm{g} / \mathrm{ml} 6.5 \pm 2 \%)$ compared to the larger-sized aSNPs of $70 \mathrm{~nm}(300 \mu \mathrm{g} / \mathrm{ml}$ : $88 \pm 18 \%, 600 \mu \mathrm{g} / \mathrm{ml}: 82 \pm 20 \%)$ and $300 \mathrm{~nm}(300 \mu \mathrm{g} /$ ml: $102 \pm 7 \%, 600 \mu \mathrm{g} / \mathrm{ml}: 102 \pm 11 \%$ ). Figure $2 \mathrm{~b}$ illustrates the LDH release after exposure to different-sized aSNPs (Sicastar Red $30 \mathrm{~nm}, 70 \mathrm{~nm}, 300 \mathrm{~nm}$ ) with an incubation period of $4 \mathrm{~h}$. In H441, LDH leakage occurred for the $30 \mathrm{~nm}$ aSNP at a concentration of $60 \mu \mathrm{g} / \mathrm{ml}(41 \pm 12 \%$ of lysis control) and reached a peak value at an aSNP concentration of $150 \mu \mathrm{g} / \mathrm{ml}(128 \pm 16 \%$ of lysis control). For $70 \mathrm{~nm}$ aSNPs, however, a marginal but still significant LDH leakage was observed at a concentration of $150 \mu \mathrm{g} / \mathrm{ml}$ (32 $\pm 14 \%$ of lysis control) and the leakage increased with augmented aSNP concentration and reached a total value at $600 \mu \mathrm{g} / \mathrm{ml}(119 \pm 11 \%$ of lysis control). Incubation with $300 \mathrm{~nm}$ aSNPs resulted in a marginal LDH release at a concentration of $600 \mu \mathrm{g} / \mathrm{ml}(35 \pm 9 \%$ of lysis control). Lower concentrations $(300-0.6 \mu \mathrm{g} / \mathrm{ml})$ did not have an effect on membrane integrity. ISO-HAS-1 showed a similar behaviour concerning MTS and LDH as the H441 after aSNP stimulation, whereat the $30 \mathrm{~nm}$ aSNPs caused a decreased viability ( $52 \pm 13 \%$ of untreated control) and an increased membrane integrity ( $84 \pm 29 \%$ of lysis control) at a concentration of $150 \mu \mathrm{g} / \mathrm{ml}$. However, the larger-sized aSNPs $(70 \mathrm{~nm})$ showed a reduced viability $(300 \mu \mathrm{g} / \mathrm{ml}: 75 \pm 12.5 \%$ and $600 \mu \mathrm{g} / \mathrm{ml}: 47 \pm 8.2 \%$ of untreated control) and an increased LDH release $(300 \mu \mathrm{g} / \mathrm{ml} ; 66 \pm 12 \%$ and 600 $\mu \mathrm{g} / \mathrm{ml}: 82 \pm 16 \%$ ) at higher concentrations. $300 \mathrm{~nm}$ aSNP did not show any significantly altered behaviour compared to the untreated control concerning MTS and LDH even at very high concentration $(600 \mu \mathrm{g} / \mathrm{ml})$.

In Fig. 3, the IL-8 release after $20 \mathrm{~h}$ further cultivation of the cells, which were exposed to aSNPs for $4 \mathrm{~h}$, is illustrated. The small-sized aSNPs $(30 \mathrm{~nm})$ caused the first peak at a concentration of $60 \mu \mathrm{g} / \mathrm{ml}$ (Fig. 3a, H441: $2.5 \pm 0.5$ and Fig. 3b, ISO-HAS-1: $2.9 \pm 0.39$-fold of untreated control, uc) whereas bigger-sized aSNP (70 nm) caused IL-8 release not until a concentration of $150 \mu \mathrm{g} / \mathrm{ml}$ (H441: $2.3 \pm 0.58$ and ISO-HAS-1: $3 \pm 0.48$-fold of uc). $300 \mathrm{~nm}$ aSNPs resulted in an IL-8 response not until a concentration of $300 \mu \mathrm{g} / \mathrm{ml}$ (H441: $1.6 \pm 0.59$ and ISOHAS-1: $2.1 \pm 0.32$-fold of uc), whereat no effects could be observed for the cytotoxicity assays (MTS, LDH) even with $600 \mu \mathrm{g} / \mathrm{ml}$. For the $30 \mathrm{~nm}$ aSNPs, the IL-8 release is decreasing (i.e. $600 \mu \mathrm{g}$ for H441: $0.47 \pm 0.2 \%$ and ISOHAS-1: $1 \pm 0.17$-fold of uc) with increasing aSNP concentration from $60 \mu \mathrm{g} / \mathrm{ml}$.

\section{Cellular uptake of different-sized aSNPs}

Figure 4 depicts the uptake and colocalization with flotillin-1/2-containing vesicles of different-sized aSNPs (30, $70,300 \mathrm{~nm}$ ). aSNPs of all sizes are incorporated in flotillin- $1 / 2$ vesicles.

aSNP exposure on flotillin-1- and flotillin-2-depleted cells

Transfection efficiency was determined via real-time PCR. After 4 days, the flotillin- 1 and flotillin-2 RNA amount was determined. Real-time PCR revealed a flotillin-1/2 RNA reduction to $32 \pm 9 \% P<0.01$ for flotillin-1 and $31 \pm 6 \%(P<0.01)$ for flotillin-2 (compared to untransfected control), whereas the negative control (neg) remained unaffected (flotillin-1: $126 \pm 17 \%$ and flotillin- $2: 93 \pm 15 \%, P>0.05$ ). In addition, the fluorescence signal of immunofluorescence-labelled flotillin1/2 decreased in flotillin-1/2-depleted cells compared to the untransfected cells (see fluorescence signals in Fig. 7). Subsequently and prior to experiments with NPs, cytotoxicity of the transfection procedure was determined via MTS. The viability of cells was not significantly affected after negative $(92 \pm 16 \%$ of untransfected control uc) and flotillin-1/2 (90 $\pm 18 \%$ of untransfected control) siRNA treatment. Four days after the transfection of $\mathrm{H} 441$ cells on 96-well plates with siRNA, cells reached a confluent growth and aSNPs (Sicastar Red) were applied to the cells for $4 \mathrm{~h}$ in serum-free medium and $4 \mathrm{~h}$ followed by 20 -h recovery in fresh FCS-containing medium. Concentrations of aSNP ranged from 6 to $300 \mu \mathrm{g} / \mathrm{ml}$. 
A

H441

$\square 30 \mathrm{~nm}=70 \mathrm{~nm} \square 300 \mathrm{~nm}$

A

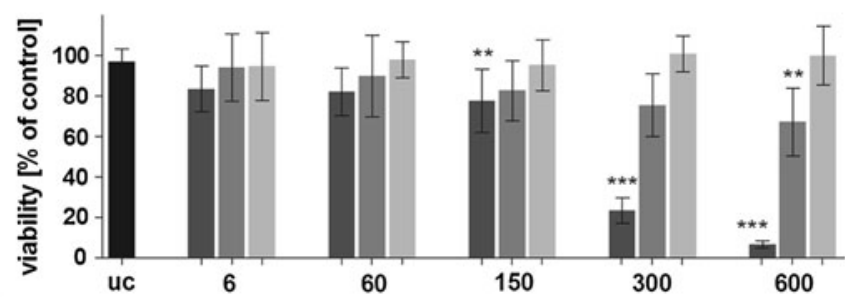

ISO-HAS-1
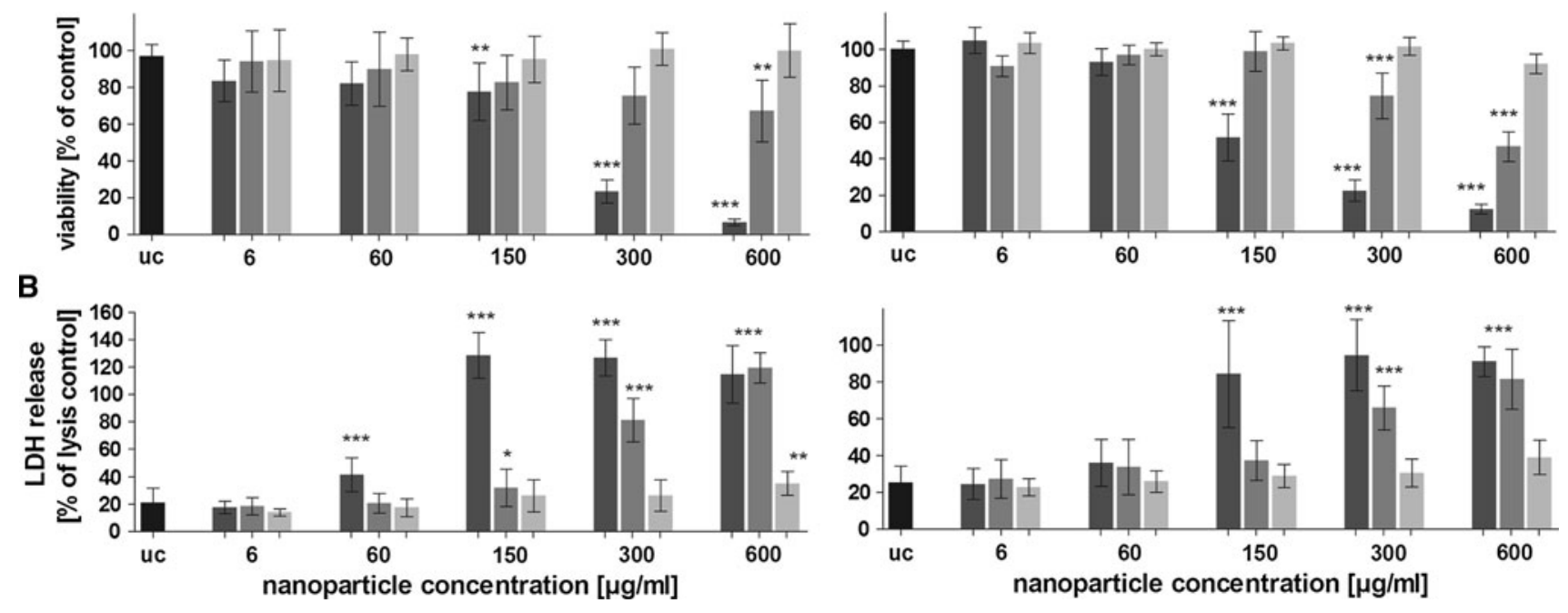

Fig. 2 a Viability (MTS assay) of H441 following aSNP treatment for $4 \mathrm{~h}$ in serum-free medium with different-sized Sicastar Red (30, $70,300 \mathrm{~nm}$ ). b Lactate dehydrogenase release (LDH) of H441 following aSNP treatment for $4 \mathrm{~h}$ with different-sized Sicastar Red $(30,70,300 \mathrm{~nm})$. Data are depicted as mean $\pm \mathrm{SD}$ of 3 independent experiments with $n=3$ samples for each treatment. For statistical

analysis, two-way ANOVA with Bonferroni's post-test was applied. $* P<0.05, * * P<0.01$ and $* * * P<0.001$ compared to the untreated control (uc). $30 \mathrm{~nm}$-sized aSNPs caused a reduced viability and an increased LDH release in H441 compared to the larger-sized aSNPs of 70 and $300 \mathrm{~nm}$

Fig. 3 The release of IL-8 was measured after aSNP (Sicastar Red, $30 \mathrm{~nm}$ : dark grey, $70 \mathrm{~nm}$ : middle grey, $300 \mathrm{~nm}$ light grey) exposure of monocultures of H441 (a) and ISO-HAS-1 (b) on 96-well plates. After 4-h incubation in serum-free medium, aSNPs were removed and the cells were cultivated for further $20 \mathrm{~h}$ in serumcontaining medium and assayed for IL-8 release. Data are depicted as mean \pm SD of $2-3$ independent experiments with $n=3$ samples for each treatment. $* P<0.05$, $* * P<0.01$ and $* * * P<0.001$ compared to the untreated control. $30 \mathrm{~nm}$-sized aSNPs caused an IL-8 response at lower aSNP concentrations in H441 and ISO-HAS-1 compared to the larger-sized aSNPs of 70 and $300 \mathrm{~nm}$
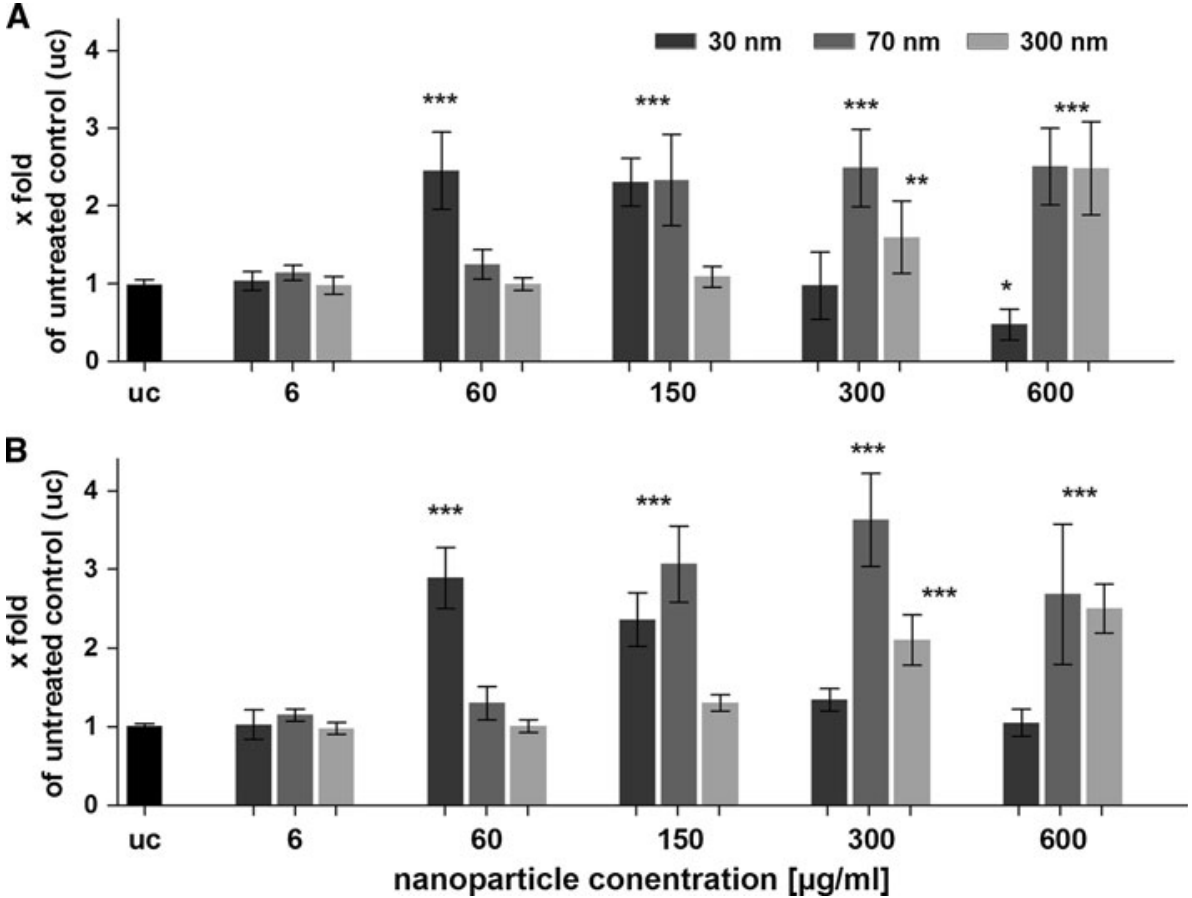

After $4 \mathrm{~h}$ of aSNP exposure, untransfected $\mathrm{H} 441$ showed a significantly decreased viability $(78 \pm 6.8 \%$ of untreated control) following Sicastar Red (30 nm) exposure at a concentration of $60 \mu \mathrm{g} / \mathrm{ml}$ (see Fig. 5). Viability further decreased with increasing concentration, and at a concentration of $300 \mu \mathrm{g} / \mathrm{ml}$, a viability of $66 \pm 5.5 \%$ was observed, compared to the untreated control. After a 20-h incubation period with fresh FCS-containing medium, the $\mathrm{H} 441$ exposed to $60 \mu \mathrm{g} / \mathrm{ml}$ aSNP "recovered" and no significant toxic effect $(95 \pm 14 \%)$ was detected. 

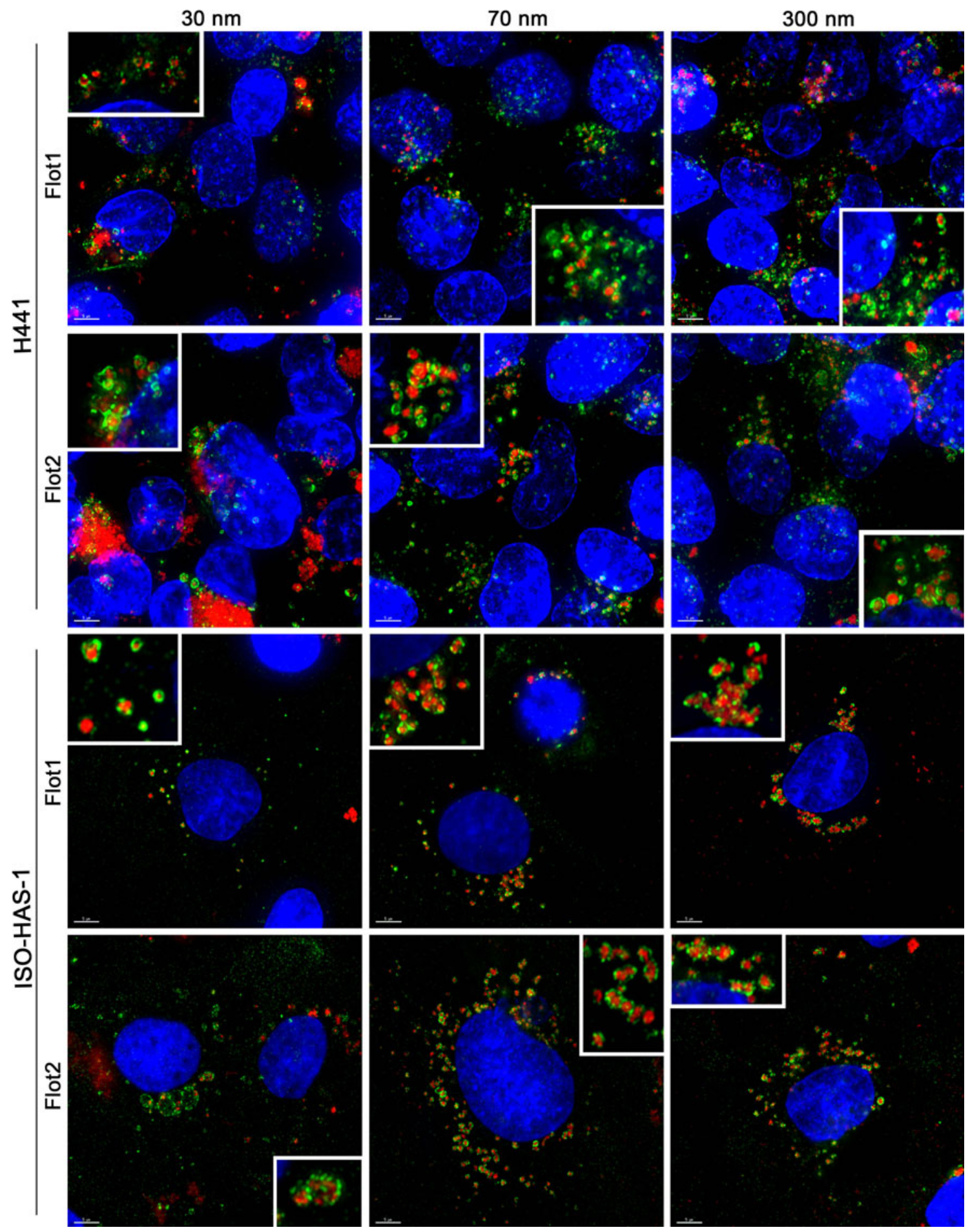

Fig. 4 Uptake studies of immunofluorescently (green signal: flotillin 1/2) stained H441 and ISO-HAS-1 kept in conventional monoculture and exposed to Sicastar Red at a concentration of $60 \mu \mathrm{g} / \mathrm{ml}(30,70$, $300 \mathrm{~nm}$ ) for $4 \mathrm{~h}$ and further 20-h cultivation (red signal). A clear incorporation of NPs in flotillin-1- and flotillin-2-containing vesicles

Concentrations of $100 \mu \mathrm{g} / \mathrm{ml}$ aSNP displayed a similar effect compared to 4-h exposure (76 \pm 13 vs. $73 \pm 9.9 \%)$, whereas $300 \mu \mathrm{g} / \mathrm{ml}$ aSNP elicited a further decline in (green signal) could be detected for all NP sizes. Nuclei are stained using Hoechst 33342 (blue). Colocalization of different-sized aSNPs in flotillin-1/2-containing vesicles occurred for all NP sizes. Scale bar $5 \mu \mathrm{m}$

viability $(28 \pm 8.8 \%)$. Comparing the viability of untransfected and transfected cells after Sicastar Red exposure, differences were detected in a concentration 
range of $6-300 \mu \mathrm{g} / \mathrm{ml}$. The non-targeted siRNA (neg) did not show any alterations compared to the untransfected cells concerning cytotoxicity after aSNP exposure. Moreover, after an incubation time of $4 \mathrm{~h}$, no significant differences between untransfected and flotillin-1/2-depleted (F12) aSNP-exposed cells were found. Nevertheless, after the 20-h recovery period, low, subtoxic concentrations of aSNPs, 6 and $60 \mu \mathrm{g} / \mathrm{ml}$, showed significant variances displaying a reduced viability of flotillin-1/2-depleted cells compared to untransfected cells (see red asterisks in Fig. 5). Figure 6 displays the IL-8 release of flotillin-1/-2depleted and aSNP-exposed $\mathrm{H} 441$ for $4 \mathrm{~h}$ with further $20-\mathrm{h}$ cultivation in fresh medium. An aSNP concentration of $100 \mu \mathrm{g} / \mathrm{ml}$ resulted in a significant IL-8 release for the untransfected control group $(2.55 \pm 0.24$-fold of untreated control with $P<0.0001$ within the untransfected control group using one-way ANOVA). For the siRNA-transfected cells, an IL-8 peak could also be detected at a concentration of $100 \mu \mathrm{g} / \mathrm{ml}$ (neg: $1.75 \pm 0.18$ and F12: $2 \pm 0.33$-fold of untreated control), but for neg significantly lower compared to the peak for the untransfected control group $(P<0.01)$ and for F12 with an increased standard deviation (0.33). Surprisingly, flotillin-1/2-depleted cells took up less aSNP (Sicastar Red) than untransfected cells according to visual assessment of images taken by a fluorescence microscope (Fig. 7). In order to obtain more specific evidence of these findings, the uptake of Sicastar Red was quantified. Since the concentrations of 6 and $60 \mu \mathrm{g} / \mathrm{ml}$ were too low to determine the amount of aSNPs taken up by $\mathrm{H} 441$ by spectrophotometric analysis, the quantification of internalized aSNP by H441 was conducted by means of intensity measurements of images made by a wide-field fluorescence microscope (personalDV, Applied Precision, Issaquah, USA). In Fig. 8 A, the relative fluorescent unit (RFU: related to the untreated control $=1$ ) was determined for the fluorescence signal of Sicastar Red, which was incubated at a concentration of $6 \mu \mathrm{g} / \mathrm{ml}$ with $\mathrm{H} 441$ in conventional monoculture. Non-targeted siRNA showed a minor, non-significant reduction of fluorescence intensity (neg: $0.64 \pm 0.17 \%$ ) of incorporated Sicastar incubated for $4 \mathrm{~h}$ followed by 20-h recovery in fresh medium. Flotillin-1/2-depleted cells, however, showed a significant decrease of internalized aSNPs according to RFU measurements (flotillin 1/2: $0.38 \pm 0.13 \%$ ) (see Fig. 8b). Furthermore, quantification studies have been conducted via immunofluorescent staining for flotillin-1/2. RFU measurements of immunofluorescent staining against flotillin-1/2 revealed a significant reduction of flotillin-1 $(0.38 \pm 0.27 \%)$ and flotillin-2 $(0.28 \pm 0.1 \%)$ fluorescence signal in flotillin-1/2-depleted H441 cells (Fig. 8a). Non-targeted siRNA transfection resulted in a minor but non-significant reduction of fluorescence intensity of flotillin-1 $(0.7 \pm 0.17 \%)$ and flotillin-2 $(0.71 \pm 0.24 \%)$. In summary, flotillin-1/2 depletion results in a reduction of flotillin-1/2 and a decreased uptake of aSNP. Furthermore, although a decreased uptake of aSNPs in flotillin-1/2depleted cells was detected, an increased toxicity of aSNPs in flotillin-1/2-depleted cells was found.

\section{Discussion}

Using MTT and LDH assays, Napierska et al. (2009) previously demonstrated a particle size-dependent cytotoxic
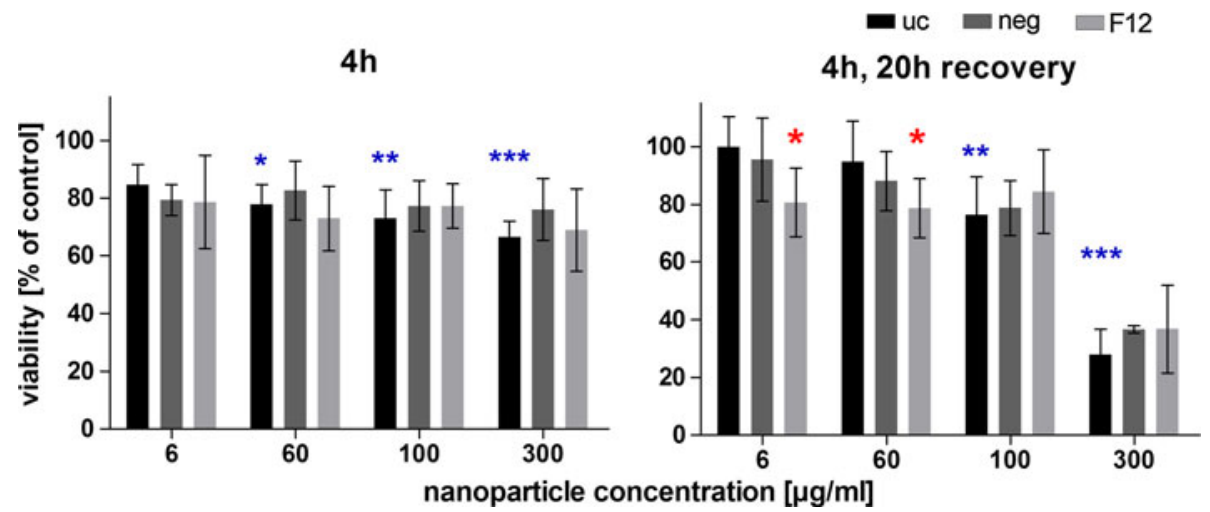

Fig. 5 Cytotoxicity (MTS) of flotillin-1- and flotillin-2-depleted H441 following aSNP treatment with $6-300 \mu \mathrm{g} / \mathrm{ml}$ Sicastar Red $(30 \mathrm{~nm}) . u c$ untransfected control (no siRNA treatment), neg Silencer ${ }^{\circledR}$ Negative Control \#1 siRNA and F12 Silencer ${ }^{\circledR}$ Select siRNA for flotillin-1 and flotillin-2 in combination (siRNA concentration: $50 \mathrm{nM}$ ). Data are depicted as mean \pm SD of 3 independent experiments with $n=3$ samples for each treatment. For statistical analysis, two-way ANOVA with Bonferroni's post-test was applied.
$* P<0.05, * * P<0.01$ and $* * * P<0.001$ compared to the untreated control of the respective siRNA pre-treatment. Blue asterisks: "oneway ANOVA: within the untransfected control", red asterisks: "twoway ANOVA: within a concentration of aSNP". After 4-h exposure and 20-h recovery, subtoxic concentrations of 6 and $60 \mu \mathrm{g} / \mathrm{ml}$ Sicastar Red showed a significantly reduced viability of flotillin-1/2-depleted cells compared to untransfected cells 


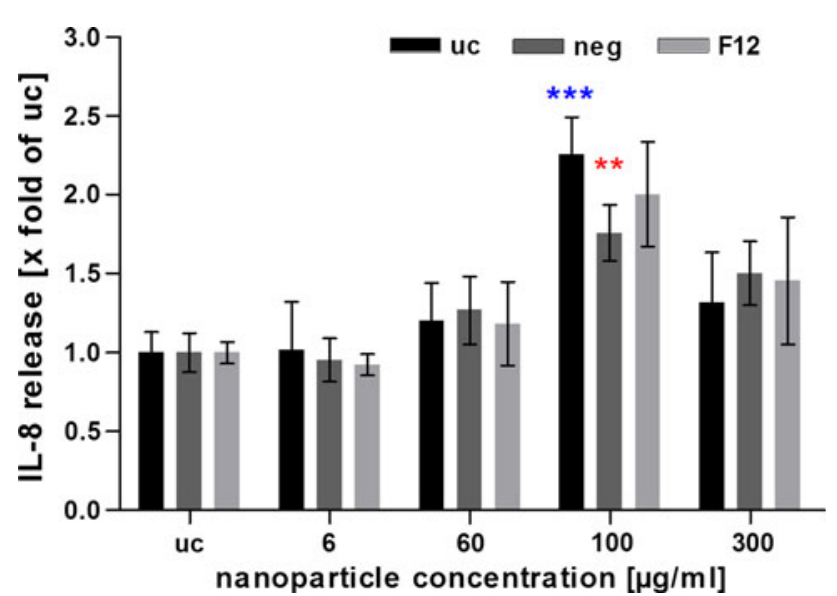

Fig. 6 IL-8 release of flotillin-1- and flotillin-2-depleted H441 following aSNP treatment with 6-300 $\mu \mathrm{g} / \mathrm{ml}$ Sicastar Red $(30 \mathrm{~nm}$ in size). $u c$ untransfected control (no siRNA treatment), neg Silencer ${ }^{\circledR}$ Negative Control \#1 siRNA and F12 Silencer ${ }^{\circledR}$ Select siRNA for flotillin-1 and flotillin-2 in combination (siRNA concentration: 50 $\mathrm{nM}$ ). Data are depicted as mean \pm SD of 2 independent experiments with $n=3$ samples for each treatment. For statistical analysis, twoway ANOVA with Bonferroni's post-test was applied. ${ }^{*} P<0.05$, $* * P<0.01$ and $* * * P<0.001$ compared to the untreated control of the respective siRNA pre-treatment. Blue asterisks: "one-way ANOVA: within the untransfected control", red asterisks: "twoway ANOVA: within a concentration of aSNP"

effect in a (supposed) human endothelial cell line (EAHY926) caused by aSNPs. The results of the present study corroborate their findings, as in this study the smaller-sized aSNPs $(30 \mathrm{~nm})$ were found to cause greater damage to lung epithelial cells (H441) as determined by the MTT and LDH assays compared to larger-sized (70, $300 \mathrm{~nm}$ ) aSNPs. Despite the fact that the $30 \mathrm{~nm}$ aSNPs showed a slight tendency of aggregation, it still displayed a higher toxicity than larger aSNPs, indicating enough nonaggregated material, which was also corroborated by the high polydispersity index $\left(\mu^{2}\left(90^{\circ}\right)=0.17\right)$. In summary, cytotoxicity appears to increase with decreasing particle size. As in Napierska et al. (2009), the dose was expressed as a mass concentration. Choosing the right dosimetry is a double-edged sword. Comparing mass concentrations of different-sized NPs leads to a comparison of different particle numbers. However, a mass concentration of, for example, $60 \mu \mathrm{g} / \mathrm{ml}$ (lowest concentration, for which a cytotoxic effect occurred with $30 \mathrm{~nm}$ aSNP) of the $30 \mathrm{~nm}$ aSNP matches with a particle number of $2 \times 10^{12}$ particles/ml according to the manufacturer's specifications, whereas $60 \mu \mathrm{g} / \mathrm{ml}$ of $300 \mathrm{~nm}$ aSNP corresponds to $2 \times 10^{9}$ particles $/ \mathrm{ml}$ (i.e. $1,000 \times$ less particles compared to $30 \mathrm{~nm}$ aSNP). However, adjusting the $300 \mathrm{~nm}$ aSNP to $2 \times 10^{12}$ particles $/ \mathrm{ml}$ would lead to a mass concentration of $60 \mathrm{mg} /$ $\mathrm{ml}$, which is far beyond any physiologically relevant amount. Additionally, the highest mass concentration of $300 \mathrm{~nm}$ that can be applied is $2 \times 10^{11}$ particles $/ \mathrm{ml}$.
However, this particle number corresponds to a mass concentration of $6 \mu \mathrm{g} / \mathrm{ml}$ for the $30 \mathrm{~nm}$ aSNPs, which is beyond the toxic range of the $30 \mathrm{~nm}$ aSNP. Moreover, an applied dose of $600 \mu \mathrm{g} / \mathrm{ml}$ and also $60 \mu \mathrm{g} / \mathrm{ml}$ does not simulate realistic exposure conditions as it might occur for, for example, a ceramic worker. These concentrations may only occur for accidently inhaled aSNP and were chosen to display the differences for the different-sized particles. Lison et al. (2008) considered mass concentration, particle number, surface area and also the applied volume of NP dispersion as crucial factors for reliable conclusions on nanoparticle cytotoxicity. In general, since the smaller the nanoparticle the bigger the surface area per mass, the surface area is supposed to be a pivotal factor for the displayed biological activity, as reviewed by Oberdörster et al. (2005).

Inflammatory cytokines such as IL-8 play a pivotal role in the recruitment and regulation of neutrophils and it has been shown by other groups to be involved in the NPinduced inflammatory reaction (Cho et al. 2007; Hetland et al. 2001). Hefland et al. (2001) also dedicated the smallest silica NPs as the most potent and correlated the inflammatory response to the surface area, since the differences between different-sized NPs disappeared when related to equal surface areas. This can be corroborated by the results of this study, whereat the IL-8 release of aSNPexposed cells (H441 and ISO-HAS-1) is increasing with decreasing particle size (when equal mass concentrations are compared). Furthermore, this study accentuated analysing inflammatory responses as the more sensitive method to examine the harmfulness of nanoparticles compared to cytotoxic assays (MTS and LDH), since significant effects could be observed at aSNP concentrations, where MTS and LDH have not yet indicated any toxic causes.

Furthermore, uptake pathways such as clathrin- and caveolae-mediated endocytosis mechanisms have been investigated via immunofluorescent staining of, for example, clathrin heavy chain, EEA1 (Early Endosome Antigen 1) or caveolin 1. However, no definite uptake of aSNPs via such conventional pathways could be detected (data not shown). However, all different-sized aSNPs were observed incorporated in flotillin-1- and flotillin-2-containing vesicles. Flotillin-1 and flotillin-2 are lipid raft proteins (also called reggie- 2 and reggie- 1 , respectively) and are discussed to be involved in clathrin- and caveolae-independent uptake pathways (Glebov et al. 2006; Ait-Slimane et al. 2009; Langhorst et al. 2008) or represent a marker for late/lysosomal compartments (Dermine et al. 2001; Glebov et al. 2006). Own observations also showed a partly colocalization of both flotillins with LAMP1 or cathepsin D in H441 and ISO-HAS-1 (data not shown).

Since the involvement of flotillins in endocytosis mechanism can be observed in a variety of cell types such 
uc

A

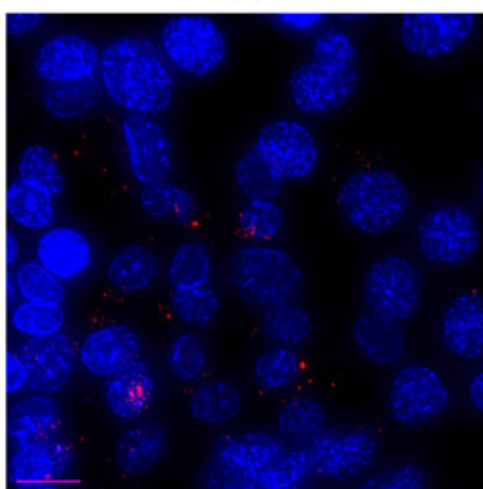

B

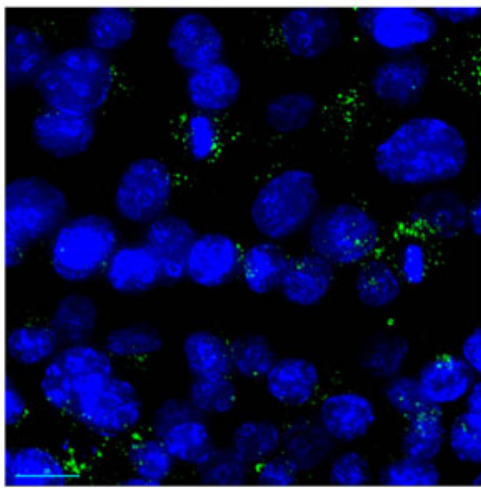

C

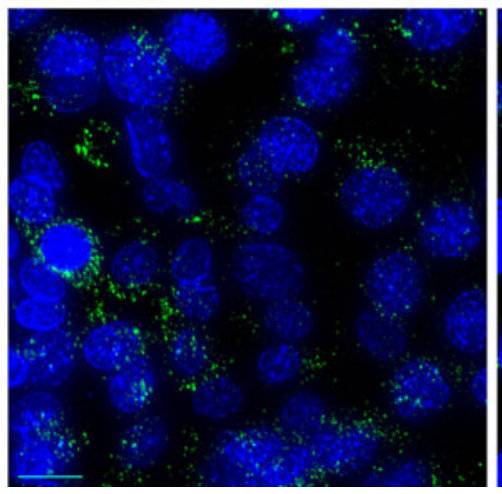

Fig. 7 a Fluorescence signal of Sicastar Red (30 nm in size, $6 \mu \mathrm{g} / \mathrm{ml})$ exposed to H441 (uc untransfected, neg non-targeted siRNA, F12 siRNA against flotillin-1/2) in conventional monoculture for $4 \mathrm{~h}$ in serum-free medium with further 20 -h cultivation in fresh serumcontaining medium. Furthermore, transfected H441 were stained via immunofluorescence for flotillin-1 (b) and flotillin-2 (c). Pictures

as macrophages (J774) (Dermine et al. 2001), epithelial cells (H441 and HeLa (Glebov et al. 2006)) or endothelial cells (ISO-HAS-1) and an incorporation of nanoparticles of different sizes (aSNP: 30, 70, $300 \mathrm{~nm}$ ) or material such as amorphous silica, organosiloxane particles or poly(ethyleneimine) (further manuscript in preparation), it appears to be a general phenomenon.

Although recent studies emphasize the size of NPs as a critical factor for entering the respective uptake pathways (Vivero-Escoto et al. 2010), in this study the size of aSNPs, ranging from 30 to $300 \mathrm{~nm}$, was not crucial for targeting neg

\section{F12}
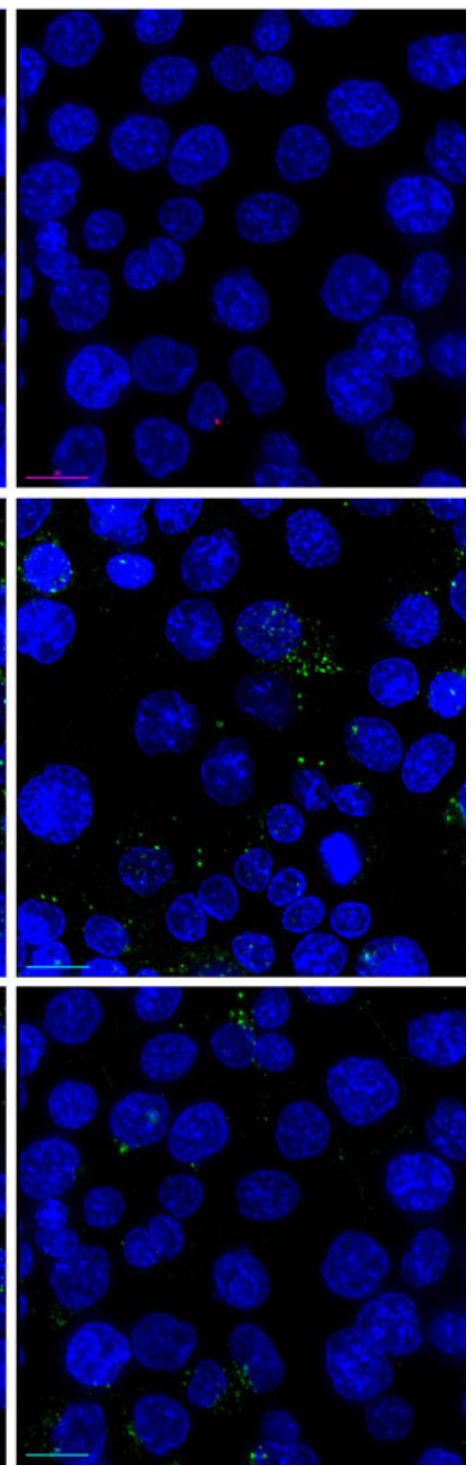

were taken by means of a fluorescence microscope (DeltaVision, Applied Precision). For appropriate comparison, exposure time and intensity scale was equally adjusted. Flotillin-1/2-depleted cells takeup less Sicastar Red than non-targeted siRNA and untransfected cells. Nuclei are stained using Hoechst 33342 (blue), scale bar $5 \mu \mathrm{m}$

flotillin-1/2-bearing vesicles in H441 and ISO-HAS-1 (see Fig. 4 for Sicastar Red after $24 \mathrm{~h}$ ). This supports the findings of Shapero and co-workers (Shapero et al. 2011), who detected an aSNP accumulation (50 and $100 \mathrm{~nm}$ in size) in lysosomes of A549 cells after $24 \mathrm{~h}$. The latter group also found a colocalization of aSNPs with early endosomes, multilamellar bodies and multivesicular bodies of A549 cells using electron microscopy, which is a more sensitive method (due to the higher resolution) to detect nanosized material than fluorescence-based detection methods, including immunofluorescence. Besides the size 
A

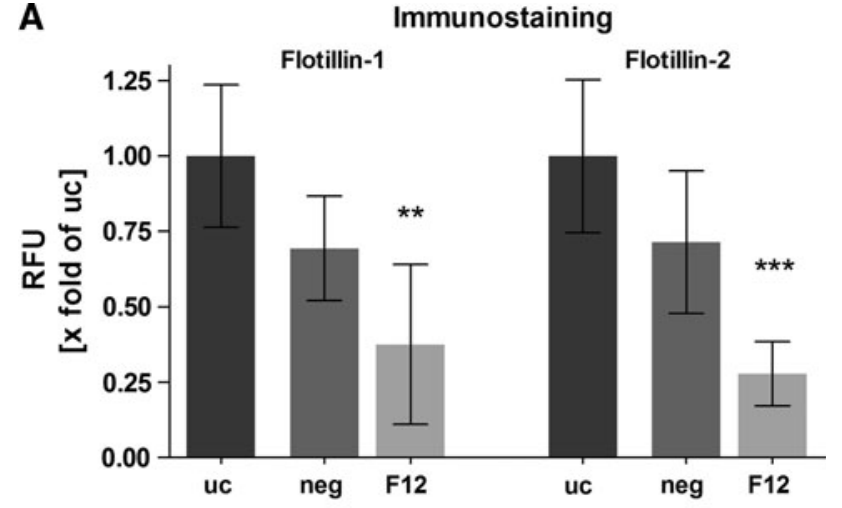

B

uptake Sicastar Red, 4h, 20h recovery

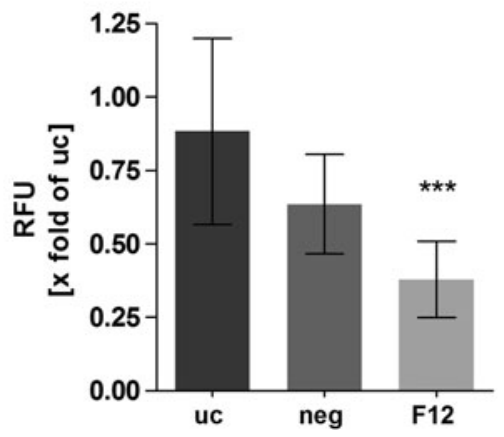

Fig. 8 Determination of RFU (relative fluorescent unit related to untransfected control cells) a Immunofluorescent staining (IF) for flotillin-1/2 and subsequent RFU measurement. Data are depicted as mean $\pm \mathrm{SD}$ of 2 independent experiments with $n=5$ images. b uptake quantification of aSNP Sicastar Red $(6 \mu \mathrm{g} / \mathrm{ml})$ in $\mathrm{H} 441$ ( $u c$ untransfected, neg treated with non-targeted siRNA, F12 Flotillin1/2-depleted cells). Exposure time: $4 \mathrm{~h}$ with further cultivation for $20 \mathrm{~h}$ in fresh medium. For statistical analysis, one-way ANOVA with Dunnett's multiple comparison test was applied with $* P<0.05$, $* * P<0.01$ and $* * * P<0.00$. Flotillin-1/2-depleted cells showed a significant reduction of flotillin-1 and flotillin-2 fluorescence signal and a decreased internalization of Sicastar Red compared to nontargeted siRNA and untransfected cells

dependency, other factors may also play a crucial role concerning cellular uptake behaviour. On the one hand, a defined surface coating of the nanoparticle is decisive, which is already indicated by several studies focussing on an enhanced uptake and retention of drugs meant for, for example, systemic or asthma treatment using surfacemodified nanovehicles (Yamamoto et al. 2005; Surti et al. 2008). On the other hand, nanoparticles, which are dispersed in protein-containing cell culture media, may adsorb proteins at the surface and form a protein corona, which is recognized by the cell despite of the NP itself (Walkey and Chan 2011).

A depletion/reduction of flotillin-1 and flotillin-2 expression in flotillin-1/2 siRNA-treated H441 cells was confirmed by real-time PCR studies. Furthermore, the fluorescence intensity of the immunofluorescent staining of flotillin-1/2 revealed a significant reduction of flotillin-1/2- containing vesicles in flotillin-1/2-depleted H441 cells. In this study, quantification of flotillin-1/2-depleted H441 cells showed a reduced uptake of Sicastar Red compared to non-transfected control cells. This finding may indicate an involvement of flotillin-1/2 in the uptake mechanisms of Sicastar Red. The control cells, which were transfected with Silencer ${ }^{\circledR}$ Negative siRNA (neg), also displayed a slight, but not significant reduction of the fluorescence intensity of the aSNP fluorescence signal. Real-time PCR results, however, did not indicate any reduction of flotillin$1 / 2$ expression in non-targeted siRNA-transfected H441. Due to the fact that the transfection procedure using Gencarrier-1, which is a lipoplex-based transfection reagent, may influence endocytosis metabolism, since the lipoplexes follow the clathrin-dependent endocytosis route, non-specific alterations in uptake of aSNPs may be explainable on the basis of these pre-treatments. For this reason, it is essential to carry out appropriate control experiments to verify non-specific influences of pre-treatments, such as transfection reagents.

The mechanisms by which aSNPs exert their cytotoxic effect are still largely unknown. However, there are many possibilities, including damage to the plasma membrane before particles penetrate the cells, intracellular interference after uptake in late or lysosomal structures, and lysosomal escape. Therefore, further experiments were conducted to obtain more insight into these possible pathways. Cytotoxicity of flotillin-1/2-depleted H441 following aSNP (Sicastar Red) exposure was evaluated and compared to non-depleted cells. The toxic influence of aSNPs (Sicastar Red) at low concentrations $(6-60 \mu \mathrm{g} / \mathrm{ml})$ was increased in flotillin-1/2-depleted cells. After $4 \mathrm{~h}$, a similar toxicity was observed for non-transfected, aSNP-treated cells and flotillin-1/2-depleted, aSNP-treated cells. However, after $20 \mathrm{~h}$ of recovery, differences between treatments became evident. While non-transfected cells appear to recover at low concentrations $(6-60 \mu \mathrm{g} / \mathrm{ml})$ within $20 \mathrm{~h}$ after exposure, the toxic effects of aSNPs persisted in flotillin-1/2-depleted cells. For higher aSNP concentrations (300 $\mu \mathrm{g} / \mathrm{ml})$, similar toxicity was observed for all cellular treatments and time-points. Taking the results of Sicastar Red uptake in flotillin-1/2-depleted H441 into account, which indicated a decelerated uptake of aSNP as seen by decreased fluorescence intensity values, an increase in cytotoxicity appears at first sight to be contradictory. Assuming an involvement of flotillins in at least storage/ degradation processes, which is corroborated by the localization of NPs in flotillin-1/2-containing vesicles, even lower concentrations of incorporated aSNP might display higher cytotoxicity in flotillin-1/2-depleted cells compared to the higher amount taken up by the control cells (nontransfected). Glebov et al. also studied the flotillin-dependent endocytosis mechanism by applying several inhibition 
methods (Damke et al. 1995; Glebov et al. 2006). They stressed the need for careful interpretation of results that are obtained from such inhibition experiments, since evidence already exists that by inhibiting a distinct endocytosis pathway, another pathway may compensate instead (Damke et al. 1995). A variety of controls have to be carried out in order to minimize other possible interpretations. This is also shown in this study, where the control group, which where transfected with Silencer ${ }^{\circledR}$ Negative siRNA, concomitant with the flotillin-1/-2 depleted cells, resulted in a significantly reduced IL-8 response after aSNP treatment compared to the untransfected, aSNP-exposed control. To approach more realistic the in vivo situation, it would be recommendable to reproduce this study with the multicellular coculture model of the alveolar-capillary barrier consisting of $\mathrm{H} 441$ on top and microvascular endothelial cells (ISO-HAS-1), which already has been used to study nanoparticle interactions (Hermanns et al. 2010; Kasper et al. 2011). However, since the H441 in coculture displays a very tight barrier with well-developed tight junctions, it is challenging to yield an efficient siRNA transfection rate, which is also shown by Hermanns et al. (2010).

In summary, this study attenuates the relevance of the nanoparticle size regarding cytotoxicity and inflammatory responses, whereat the smaller the size of the nanoparticle is, the more potent it causes harmful effects. Furthermore, all different aSNPs sizes have been incorporated in flotillin-1- and flotillin-2-labelled vesicles in lung epithelial and endothelial cells, which display a marker for late endosomal or lysosomal structures and appear to exhibit a clathrin- or caveolae-independent mode of endocytosis. Flotillin-depleted epithelial cells showed a clearly decreased uptake of aSNPs. Additionally, the viability of cells after aSNP exposure was reduced in these cells. These findings indicate a contribution of flotillins in as yet unknown (clathrin or caveolae independent) endocytosis mechanisms and (or) endosomal storage.

\begin{abstract}
Acknowledgments A portion of the work described herein was carried out by Jennifer Kasper in partial fulfilment of the requirements for a biological doctoral degree at the Johannes Gutenberg University, Mainz, Germany. The authors wish to thank Ms Elke Hübsch and Ms Michaela Moisch for their excellent assistance with the cell culture and immunocytochemical studies. This study was supported by the DFG priority program SPP 1313 within the Cluster BIONEERS and also by the European Union, FP6 Project NanoBioPharmaceutics.
\end{abstract}

Conflict of interest The authors declare that they have no competing interests.

Open Access This article is distributed under the terms of the Creative Commons Attribution License which permits any use, distribution, and reproduction in any medium, provided the original author(s) and the source are credited.

\section{References}

Ait-Slimane T, Galmes R, Trugnan G, Maurice M (2009) Basolateral internalization of GPI-anchored proteins occurs via a clathrinindependent flotillin-dependent pathway in polarized hepatic cells. Mol Biol Cell 20(17):3792-3800. doi:10.1091/mbc.E0904-0275

Bailey MM, Berkland CJ (2009) Nanoparticle formulations in pulmonary drug delivery. Med Res Rev 29(1):196-212. doi: $10.1002 /$ med.20140

Borm PJ, Robbins D, Haubold S, Kuhlbusch T, Fissan H, Donaldson K, Schins R, Stone V, Kreyling W, Lademann J, Krutmann J, Warheit D, Oberdorster E (2006) The potential risks of nanomaterials: a review carried out for ECETOC. Part Fibre Toxicol 3:11. doi:10.1186/1743-8977-3-11

Cho WS, Choi M, Han BS, Cho M, Oh J, Park K, Kim SJ, Kim SH, Jeong J (2007) Inflammatory mediators induced by intratracheal instillation of ultrafine amorphous silica particles. Toxicol Lett 175(1-3):24-33

Damke H, Baba T, van der Bliek AM, Schmid SL (1995) Clathrinindependent pinocytosis is induced in cells overexpressing a temperature-sensitive mutant of dynamin. J Cell Biol 131(1): 69-80

Dermine JF, Duclos S, Garin J, St-Louis F, Rea S, Parton RG, Desjardins M (2001) Flotillin-1-enriched lipid raft domains accumulate on maturing phagosomes. J Biol Chem 276(21):18507-18512. doi:10.1074/jbc.M101113200

Ellsworth DK, Verhulst D, Spitler TM, Sabacky BJ (2000) Titanium nanoparticles move to the marketplace. Chem Innov 30(12): $30-35$

Glebov OO, Bright NA, Nichols BJ (2006) Flotillin-1 defines a clathrin-independent endocytic pathway in mammalian cells. Nat Cell Biol 8(1):46-54. doi:10.1038/ncb1342

Hermanns MI, Kasper J, Dubruel P, Pohl C, Uboldi C, Vermeersch V, Fuchs S, Unger RE, Kirkpatrick CJ (2010) An impaired alveolarcapillary barrier in vitro: effect of proinflammatory cytokines and consequences on nanocarrier interaction. J R Soc Interface 7(suppl 1):S41-S54. doi:10.1098/rsif.2009.0288.focus

Hetland RB, Schwarze PE, Johansen BV, Myran T, Uthus N, Refsnes M (2001) Silica-induced cytokine release from A549 cells: importance of surface area versus size. Hum Exp Toxicol 20(1): $46-55$

Hoet PH, Bruske-Hohlfeld I, Salata OV (2004) Nanoparticlesknown and unknown health risks. J Nanobiotechnology 2(1):12. doi:10.1186/1477-3155-2-12

Institute of Nanotechnology hwnou (2011) Institute of Nanotechnology, http://www.nano.org.uk/

Kasper J, Hermanns MI, Bantz C, Maskos M, Stauber R, Pohl C, Unger RE, Kirkpatrick JC (2011) Inflammatory and cytotoxic responses of an alveolar-capillary coculture model to silica nanoparticles: comparison with conventional monocultures. Part Fibre Toxicol 8(1):6. doi:10.1186/1743-8977-8-6

Kawashima Y, Yamamoto H, Takeuchi H, Fujioka S, Hino T (1999) Pulmonary delivery of insulin with nebulized DL-lactide/glycolide copolymer (PLGA) nanospheres to prolong hypoglycemic effect. J Control Release 62(1-2):279-287. doi:S0168-3659(99) 00048-6 [pii]

Kreyling WG, Semmler-Behnke M, Chaudhry Q (2010) A complementary definition of nanomaterial. Nano Today 5(3):165-168. doi:10.1016/j.nantod.2010.03.004

Langhorst MF, Reuter A, Jaeger FA, Wippich FM, Luxenhofer G, Plattner H, Stuermer CA (2008) Trafficking of the microdomain scaffolding protein reggie-1/flotillin-2. Eur J Cell Biol 87(4): 211-226. doi:10.1016/j.ejcb.2007.12.001 
Li Y, Sun L, Jin M, Du Z, Liu X, Guo C, Huang P, Sun Z (2011) Sizedependent cytotoxicity of amorphous silica nanoparticles in human hepatoma HepG2 cells. Toxicol In Vitro. doi:10.1016/ j.tiv.2011.05.003

Lison D, Thomassen LC, Rabolli V, Gonzalez L, Napierska D, Seo JW, Kirsch-Volders M, Hoet P, Kirschhock CE, Martens JA (2008) Nominal and effective dosimetry of silica nanoparticles in cytotoxicity assays. Toxicol Sci 104(1):155-162

Liu Y, Lou C, Yang H, Shi M, Miyoshi H (2011) Silica nanoparticles as promising drug/gene delivery carriers and fluorescent nanoprobes: recent advances. Curr Cancer Drug Targets 11(2): 156-163. doi:EPub-Abstract-CCDT-96 [pii]

Masuzawa M, Fujimura T, Hamada Y, Fujita Y, Hara H, Nishiyama S, Katsuoka K, Tamauchi H, Sakurai Y (1999) Establishment of a human hemangiosarcoma cell line (ISO-HAS). Int $\mathrm{J}$ Cancer 81(2):305-308

Nabeshi H, Yoshikawa T, Arimori A, Yoshida T, Tochigi S, Hirai T, Akase T, Nagano K, Abe Y, Kamada H, Tsunoda S, Itoh N, Yoshioka Y, Tsutsumi Y (2011) Effect of surface properties of silica nanoparticles on their cytotoxicity and cellular distribution in murine macrophages. Nanoscale Res Lett 6(1):93. doi: 10.1186/1556-276X-6-93

Napierska D, Thomassen LCJ, Rabolli V, Lison D, Gonzalez L, KirschVolders M, Martens JA, Hoet PH (2009) Size-dependent cytotoxicity of monodisperse silica nanoparticles in human endothelial cells. Small 5(7):846-853. doi:10.1002/smll.200800461

Oberdorster G, Oberdorster E, Oberdorster J (2005) Nanotoxicology: an emerging discipline evolving from studies of ultrafine particles. Environ Health Perspect 113(7):823-839

Service RF (2004) Nanotoxicology: nanotechnology grows up. Science 304(5678):1732-1734

Shapero K, Fenaroli F, Lynch I, Cottell DC, Salvati A, Dawson KA (2011) Time and space resolved uptake study of silica nanoparticles by human cells. Mol BioSyst 7(2):371-378. doi: 10.1039/c0mb00109k

Sun L, Li Y, Liu X, Jin M, Zhang L, Du Z, Guo C, Huang P, Sun Z (2011) Cytotoxicity and mitochondrial damage caused by silica nanoparticles. Toxicol In Vitro. doi:10.1016/j.tiv.2011.06.012

Surti N, Naik S, Bagchi T, Dwarkanath BS, Misra A (2008) Intracellular delivery of nanoparticles of an antiasthmatic drug. AAPS PharmSciTech 9(1):217-223. doi:10.1208/s12249-0089036- $x$

Unger RE, Krump-Konvalinkova V, Peters K, Kirkpatrick CJ (2002) In vitro expression of the endothelial phenotype: comparative study of primary isolated cells and cell lines, including the novel cell line HPMEC-ST1.6R. Microvasc Res 64(3):384-397

Vivero-Escoto JL, Slowing II, Trewyn BG, Lin VS (2010) Mesoporous silica nanoparticles for intracellular controlled drug delivery. Small 6(18):1952-1967. doi:10.1002/smll.200901789

Walkey CD, Chan WC (2011) Understanding and controlling the interaction of nanomaterials with proteins in a physiological environment. Chem Soc Rev. doi:10.1039/c1cs15233e

Waters KM, Masiello LM, Zangar RC, Tarasevich BJ, Karin NJ, Quesenberry RD, Bandyopadhyay S, Teeguarden JG, Pounds JG, Thrall BD (2009) Macrophage responses to silica nanoparticles are highly conserved across particle sizes. Toxicol Sci 107(2): 553-569

Yamamoto H, Kuno Y, Sugimoto S, Takeuchi H, Kawashima Y (2005) Surface-modified PLGA nanosphere with chitosan improved pulmonary delivery of calcitonin by mucoadhesion and opening of the intercellular tight junctions. J Control Release 102(2):373-381. doi:10.1016/j.jconrel.2004.10.010

Zhang Q, Shen Z, Nagai T (2001) Prolonged hypoglycemic effect of insulin-loaded polybutylcyanoacrylate nanoparticles after pulmonary administration to normal rats. Int $\mathbf{J}$ Pharm 218(1-2): 75-80. doi:S0378-5173(01)00614-7 [pii] 